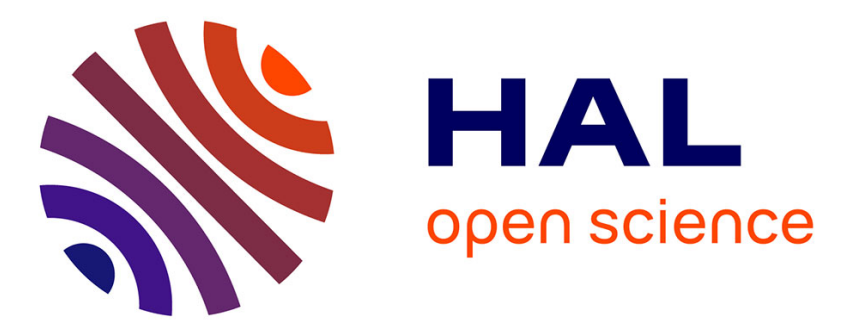

\title{
Electronic Properties of WS 2 /WSe 2 Heterostructure Containing Te Impurity: The Role of Substituting Position
}

\author{
A. Krivosheeva, V. Shaposhnikov, V. e. Borisenko, J.-L. Lazzari
}

\section{- To cite this version:}

A. Krivosheeva, V. Shaposhnikov, V. e. Borisenko, J.-L. Lazzari. Electronic Properties of WS 2 /WSe 2 Heterostructure Containing Te Impurity: The Role of Substituting Position. International Journal of Nanoscience, 2019, 18 (03n04), pp.1940007. 10.1142/S0219581X19400076 . hal-03144479

\author{
HAL Id: hal-03144479 \\ https://hal.science/hal-03144479
}

Submitted on 17 Feb 2021

HAL is a multi-disciplinary open access archive for the deposit and dissemination of scientific research documents, whether they are published or not. The documents may come from teaching and research institutions in France or abroad, or from public or private research centers.
L'archive ouverte pluridisciplinaire HAL, est destinée au dépôt et à la diffusion de documents scientifiques de niveau recherche, publiés ou non, émanant des établissements d'enseignement et de recherche français ou étrangers, des laboratoires publics ou privés. 


\section{Accepted Manuscript}

\section{International Journal of Nanoscience}

Article Title:

Author(s):

DOI:

Received:

Accepted:

To be cited as:

Link to final version:
Electronic Properties of $\mathrm{WS}_{2} / \mathrm{WSe}_{2}$ Heterostructure Containing Te Impurity: The Role of Substituting Position

A. V. Krivosheeva, V. L. Shaposhnikov, V. E. Borisenko, J.-L. Lazzari

$10.1142 / \mathrm{S} 0219581 \mathrm{X} 19400076$

22 December 2018

08 February 2019

A. V. Krivosheeva et al., Electronic Properties of $\mathrm{WS}_{2} / \mathrm{WSe}_{2}$ Heterostructure Containing Te Impurity: The Role of Substituting Position, International Journal of Nanoscience, doi: 10.1142/S0219581X19400076

https://doi.org/10.1142/S0219581X19400076

This is an unedited version of the accepted manuscript scheduled for publication. It has been uploaded in advance for the benefit of our customers. The manuscript will be copyedited, typeset and proofread before it is released in the final form. As a result, the published copy may differ from the unedited version. Readers should obtain the final version from the above link when it is published. The authors are responsible for the content of this Accepted Article. 


\title{
ELECTRONIC PROPERTIES OF WS /WSe $_{2}$ HETEROSTRUCTURE CONTAINING Te IMPURITY: THE ROLE OF SUBSTITUTING POSITION
}

\author{
A. V. Krivosheeva ${ }^{1}$, V. L. Shaposhnikov ${ }^{1}$, V. E. Borisenko ${ }^{1,2}$ \\ ${ }^{1}$ Belarusian State University of Informatics and Radioelectronics, P. Browka 6, 220013 Minsk, Belarus \\ ${ }^{2}$ National Research Nuclear University MEPhI, 115409 Moscow, Kashirskoe Shosse 31, Russia \\ anna@nano.bsuir.edu.by \\ J.-L. Lazzari \\ Aix-Marseille Université, CNRS, CINaM, Marseille, France
}

Received 22 December 2018; accepted 8 February 2019

\begin{abstract}
An impact of the positions of Te atoms substituting $\mathrm{W}$ atoms in two-dimensional $\mathrm{WS}_{2} / \mathrm{WSe}_{2}$ heterostructures on their electronic properties is investigated by theoretical simulation. The substitution of $\mathrm{W}$ by Te tends to reduce the energy band gap and can lead to metallic properties depending on the impurity position and concentration.
\end{abstract}

Keywords: 2D heterostructure, dichalcogenide, electronic property, impurity, substituting position.

\section{Introduction}

Individual layers of transition metal dichalcogenides, contrary to their bulk form, are known to possess direct-gap properties. ${ }^{1-4}$ Analogously, stacking of two monolayers (MLs) of different dichalcogenides may also provide the direct nature of the gap. Moreover, doping may change band gap values and related characteristics of such heterostructures. In order to investigate how Te atoms can influence on electronic properties of a two-dimensional heterostructure made of $\mathrm{WS}_{2}$ and $\mathrm{WSe}_{2} \mathrm{MLs}$, we have calculated electronic energy band structures of different variants of the Te atoms substituting metal atoms in such structures and compared with our previous results for the case when $\mathrm{Te}$ atoms substituted the chalcogen ones. ${ }^{5}$ An impact of Te atomic positions was analyzed.

\section{Method}

The heterostructure considered was composed of MLs of two different materials, namely $\mathrm{WS}_{2}$ and $\mathrm{WSe}_{2}$. We analyzed $2 \mathrm{H}$ phase with the space group $\mathrm{P}_{3} / m m c$ as the most stable polymorphic modification for this class of hexagonal layered two-dimensional crystals. ${ }^{6}$ The layers were arranged in accordance with the bulk material. Electronic energy band structure and densities of states (DOSs) for $\mathrm{WS}_{2} / \mathrm{WSe}_{2}$ heterostructure were calculated using the PAW-LDA approximation $^{7}$ within the density functional theory realized in the VASP code. ${ }^{8}$ Characteristics of the heterostructures were modeled using optB86b-vdW optimized exchange functional of J. Klimeš, ${ }^{9}$ describing the van der Waals interaction between the monolayers, implemented in VASP code, which gives the structural parameters very close to experimental values.

We used $2 \times 2$ translational cell. The thickness of the vacuum spacer between the repeating layers was $15 \AA$. Three different positions of $\mathrm{Te}$ atoms were considered as illustrated in Fig. 1: 1) one Te atom in $\mathrm{WS}_{2}$ layer; 2) one Te atom in $\mathrm{WSe}_{2}$ layer; 3 ) Te atoms in both layers. These compositions correspond to 25 at.\%, 25 at.\%, and 50 at.\% of Te concentrations, respectively. 

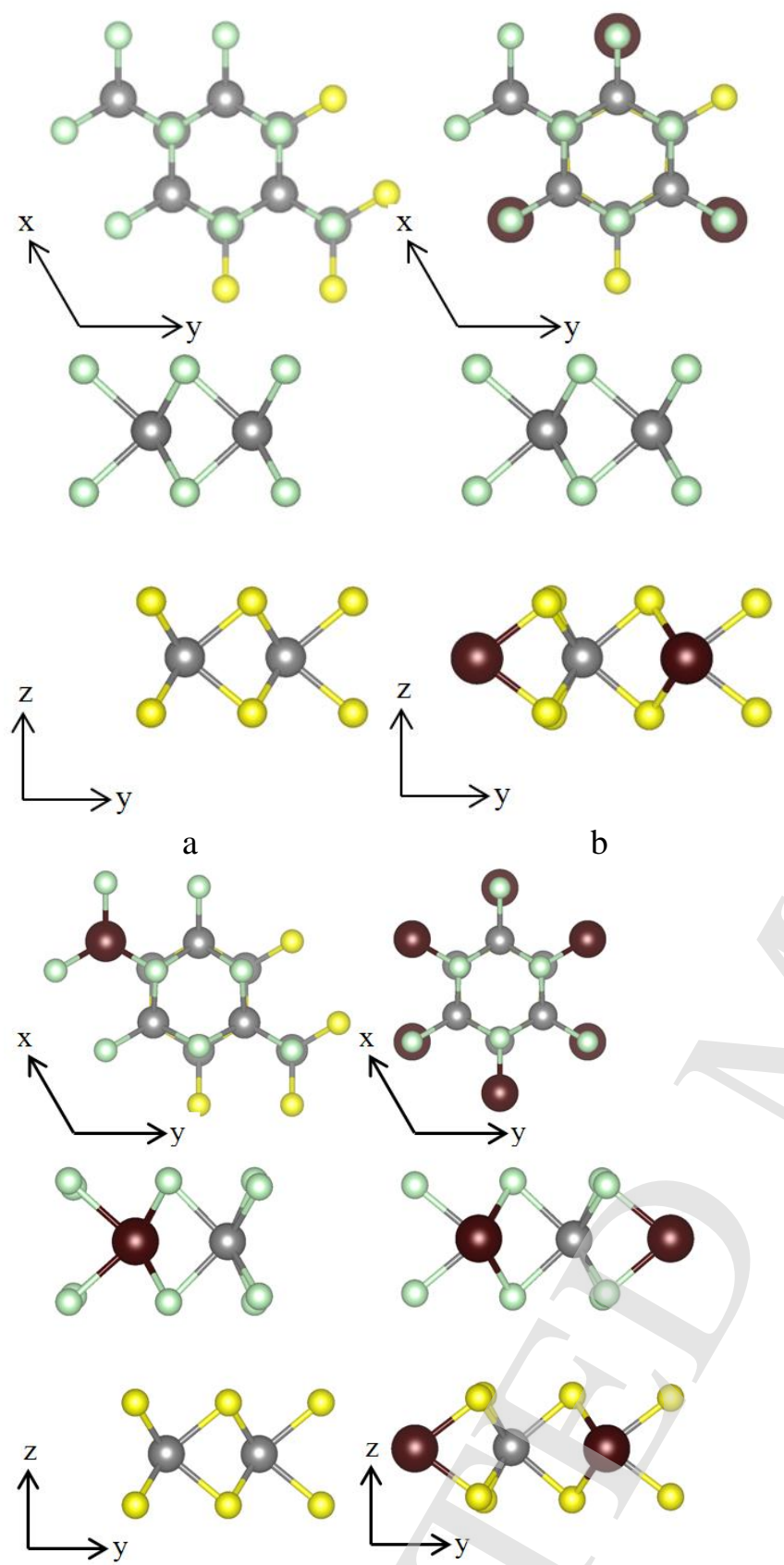

$\mathrm{c}$

d

Fig. 1. Top and side view of $\mathrm{WS}_{2} / \mathrm{WSe}_{2}$ heterostructure (a) and the heterostructure with three different positions of Te atoms (b-d). Dark small balls represent $\mathrm{W}$ atoms, light small balls stand for chalcogen atoms ( $\mathrm{S}$ atoms in bottom layer, Se atoms in top layer), large dark balls represent Te atoms.

Atomic relaxation via minimization of the total energy of the heterostructures was stopped when the forces acting on atoms became less than $0.01 \mathrm{eV} / \AA$. The energy cutoff was fixed at $380 \mathrm{eV}$. The $9 \times 9 \times 2$ $\Gamma$-centered grid of k-points was used.

\section{Results and Discussion}

The electronic band structures of $\mathrm{WS}_{2} / \mathrm{WSe}_{2}$ heterostructure and total DOSs for all the cases considered are presented in Fig. 2 and Fig. 3, respectively. It is obvious, that substitution of $\mathrm{W}$ atoms by $\mathrm{Te}$ ones strictly reduces the band gap, leading to an appearance of the electronic states at the Fermi level when Te substitutes $\mathrm{Se}$ and, thus, to a metallic behavior. This differs from the variant, when chalcogen atom was substituted, as described in our previous paper. ${ }^{5}$ In that case the gap was reduced as well, but the heterostructure preserved its semiconductor properties.
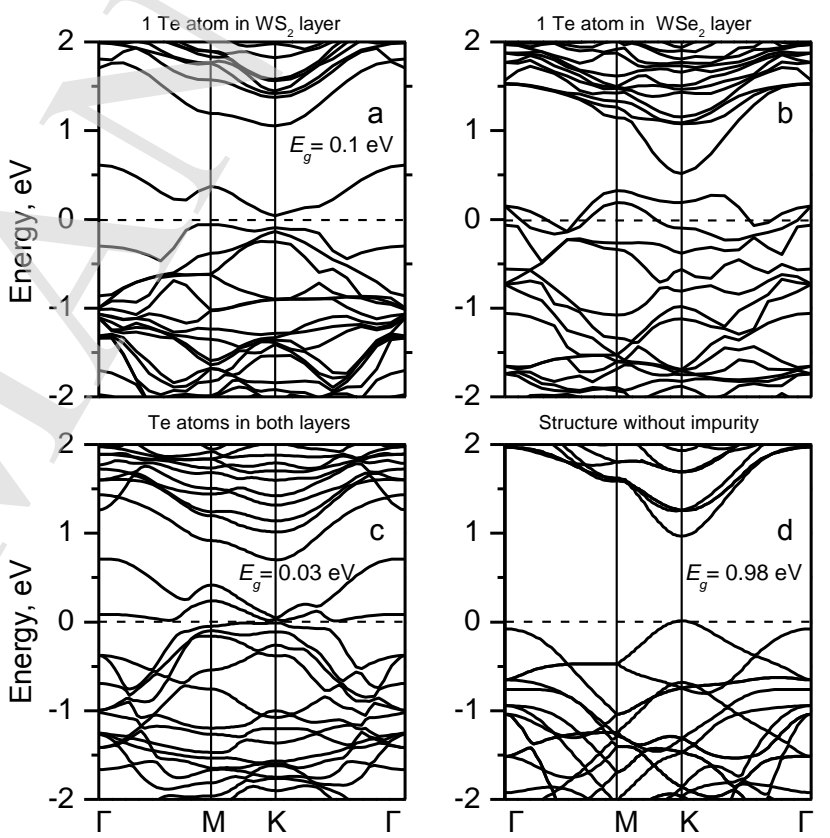

Fig. 2. Electronic energy bands in $\mathrm{WS}_{2} / \mathrm{WSe}_{2}$ heterostructures with $(a, b, c)$ and without (d) Te atoms substituting $\mathrm{W}$ ones. Zero on the energy scale corresponds to the Fermi level.

The partial DOSs of undoped $\mathrm{WS}_{2} / \mathrm{WSe}_{2}$ heterostructures are presented in Fig. 3. The main impact on the band forming states is determined by $d$-states of $\mathrm{W}$ and $p$-states of chalcogen atoms ( $s$-states are not shown).

Calculation of partial DOSs (not shown here) gave us a detailed information how the states are redistributed upon the substitution of $\mathrm{W}$ atoms by $\mathrm{Te}$ ones. When Te atom is located in $\mathrm{WS}_{2}$ layer, the states near the Fermi level are determined by $d$-electrons of $\mathrm{W}$ and $p$-electrons of $\mathrm{S}$. The substitution of $\mathrm{W}$ atom by $\mathrm{Te}$ one in $\mathrm{WSe}_{2}$ layer leads to an appearance of $p$-electrons of Se and $d$-electrons of $\mathrm{W}$ at the Fermi 
level and, thus, to transformation of the semiconducting material to a metal.

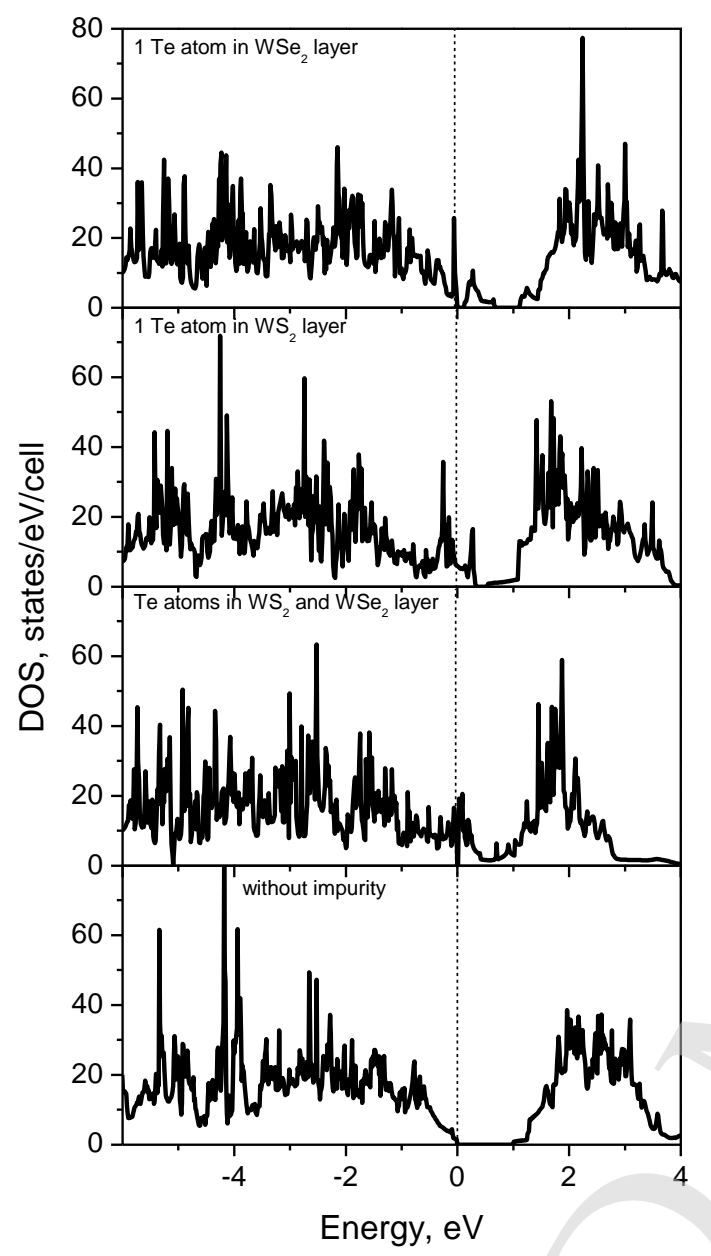

Fig. 3. Total DOSs of $\mathrm{WS}_{2} / \mathrm{WSe}_{2}$ heterostructures with $(\mathrm{a}, \mathrm{b}, \mathrm{c})$ and without (d) Te impurity. Zero on the energy scale corresponds to the Fermi level.

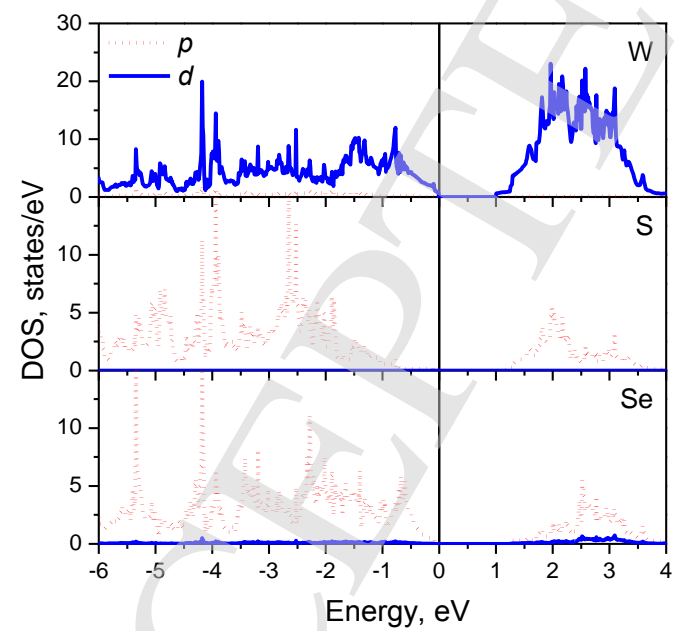

Fig. 4. Partial DOSs of undoped $\mathrm{WS}_{2} / \mathrm{WSe}_{2}$ heterostructures. Zero on the energy scale corresponds to the Fermi level.
However, the most interesting thing occurs, when Te atoms are located in both layers. We observe the appearance of the energy gap and recovery of semiconducting properties (Fig. 2c), whereas the states near the Fermi level are determined by $p$-electrons of both $\mathrm{S}$ and $\mathrm{Se}$ atoms. Contribution of Te atoms in the gap forming states is quite negligible in all the cases and is well-observed only for far-lying states.

\section{Conclusion}

Substitution of $\mathrm{W}$ atoms by Te ones in $\mathrm{WS}_{2}$ layer in $\mathrm{WS}_{2} / \mathrm{WSe}_{2}$ heterostructure strictly reduces its energy band gap, but preserves the semiconducting properties. Analogously, the heterostructure with Te atoms both in $\mathrm{WS}_{2}$ and $\mathrm{WSe}_{2}$ layers possesses a small band gap, whereas the substitution of $\mathrm{W}$ atoms by Te ones only in $\mathrm{WSe}_{2}$ layer leads to an appearance of new states at the Fermi level and to metallic properties.

\section{Acknowledgments}

This work was supported by the project No F17MC-017 of BRFFR and Belarusian State Scientific Program "Functional and Engineering Materials, Nanomaterials".

\section{References}

1. K. F. Mak, C. Lee, J. Hone, J. Shan, T. F. Heinz, Phys. Rev. Lett. 105, 136805 (2010).

2. A. Splendiani, L.Sun, Y. Zhang, Y. Li, J. Kim, C. Y. Chim, G. Galli, F. Wang, Nano Letters 10, 1271 (2010).

3. A. V. Krivosheeva, $\quad$ V. L. Shaposhnikov, V. E. Borisenko, J.-L. Lazzari, N. V. Skorodumova, B. K. Tay, Int. J. Nanotechnol. 12, 654 (2015).

4. A. V. Krivosheeva, V. L. Shaposhnikov, V. E. Borisenko, J.-L. Lazzari, C. Waileong, J. Gusakova, B. K. Tay, J. Semicond. 36, 122002 (2015).

5. V. L. Shaposhnikov, $\quad$ A. V. Krivosheeva, V. E. Borisenko, Phys. Stat. Sol. B, 1800355 (2019).

6. R. Evarestov, A. Bandura, V. Porsev, A. Kovalenko, J. Comput. Chem. 38, 2581 (2017).

7. D. M. Ceperley, B. J. Alder, Phys. Rev. Lett. 45, 566 (1980).

8. G. Kresse, J. Furthmüller, Phys. Rev. B 54, 11169 (1996).

9. J. Klimeš, D. R. Bowler, A. Michaelides, Phys. Rev. B 83, 195131 (2011). 